\title{
Use of bedside ultrasound by non-radiologists for the rapid diagnosis of life threatening conditions
}

\author{
Fonseka CL, Singhapura SDAL, Jayasekara JMS, Wijayarathna WMCJB, Kularatne OAR, de Silva \\ CM, Dissanayake AS
}

Professorial Medical Unit, Teaching Hospital, Karapitiya, Galle, Sri Lanka.

Correspondence: Dr. C LFonseka

e-mail: fonseka.lakmal@gmail.com

\section{Case Report}

A 39-year old female with a history of Type 2 Diabetes for the last two years was admitted with progressively worsening shortness of breath, nonproductive cough and right sided pleuritic-type chest pain of two-days. She was a non-smoker and denied history of fever, night sweats, or tuberculosis exposure. She had lethargy, loss of appetite and unexplained loss of weight over the last month. She denied a history of haemoptysis, orthopnoea, paroxysmal nocturnal dyspnea or leg swelling.

On examination, she was average built and afebrile. However, she was dyspnoeic with respiration rate of 28 breaths per minute and peripheral oxygen saturation was $95 \%$ on room air. She was not pale and her heart rate was 126 beats per minute with blood pressure of 115/78 $\mathrm{mmHg}$. Her jugular venous pressure was not elevated but her heart sounds were soft and no pericardial friction rub could be heard. There was a right sided moderate pleural effusion with no evidence of mediastinal shift. No cervical or generalised lymphadenopathy or organomegaly observed.

The possibility of tuberculosis or malignancy complicated with pleural and pericardial effusions was considered. ECG showed sinus tachycardia with low voltage complexes. Urgent inward ultrasound scan (Figure 1) done by junior doctors in the medical team showed a significant pericardial effusion with mild right atrial collapse. The inferior vena cava was non collapsible.

Initial investigations revealed normal blood counts and blood picture showed no abnormality. Initial ESR was 32 in the first hour and LDH was
$852 \mathrm{U} / \mathrm{L}$ (normal 230-450). Arterial blood gas analysis showed an oxygen partial pressure of 85 $\mathrm{mmHg}$ with normal $\mathrm{HCO}_{3}$ and lactate level. Chest radiograph revealed right sided moderate size pleural effusion and widening of the mediastinum with widened cardiothoracic index (Figure 2). Mantoux test was negative and no Acid Fast Bacilli were isolated in sputum.

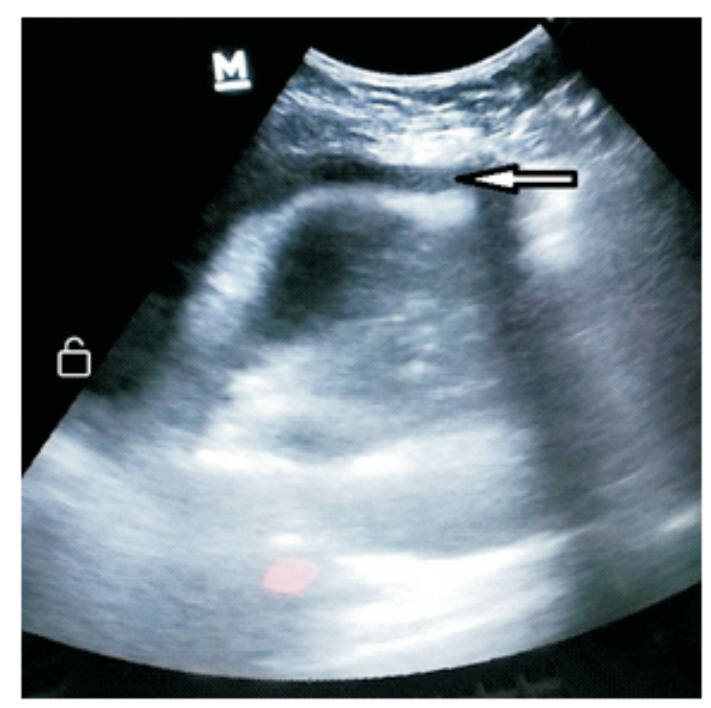

Figure 1: Bedside ultrasound scan showing evidence of a pericardial effusion

Based on US scan findings, the possibility cardiac tamponade was suspected and urgent cardiology referral was done. 2D Echocardiogram revealed a large pericardial effusion (size $8 \mathrm{~mm}$ anteriorly) with extrinsic compression of the right atrium and a mixed echogenic mass. An urgent pericardial aspirate was performed and $120 \mathrm{ml}$ fluid was 
removed. Pericardial sheath was kept in-situ in order to aspirate the rapidly filling effusion. Pericardial and pleural aspirates were blood stained and had negative cultures and showed no malignant cells. CECT chest and abdomen revealed an anterior mediastinal mass, most likely a non-Hodgkin's lymphoma (Figure 3). Mediastinal biopsy confirmed the diagnosis of high grade Non-Hodgkin's Lymphoma (NHL) and the patient was referred for chemotherapy and oncology follow-up.

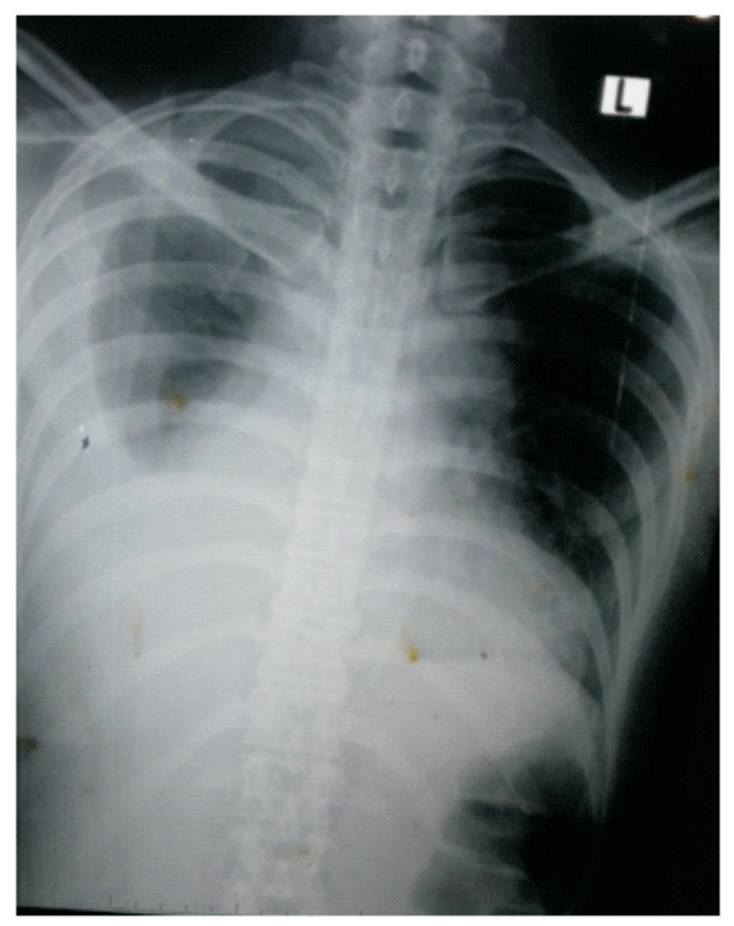

Figure 2: Chest radiograph showing right sided moderate pleural effusion with wide mediastinum

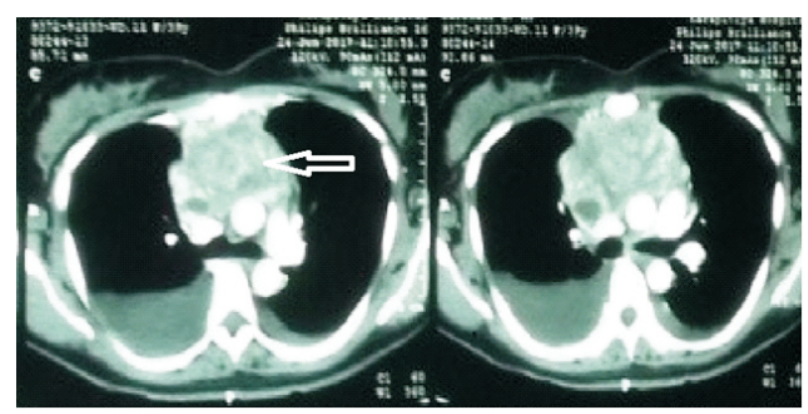

Figure 3: CECT Chest showing a heterogenous anterior mediastinal mass

\section{Discussion}

In lymphoma, either non-Hodgkin's or Hodgkin's, the frequency of pleural effusion is around 20-30\%, and the involvement of peritoneal and pericardial cavities is usually uncommon (1). But, in advanced stage III and IV NHL patients, echocardiographic examination has revealed the presence of pericardial effusion in nearly half of patients (2). Pericardial effusion due to a malignant anterior mediastinal mass could occur due to direct invasion of the pericardium and / or haematogenous spread. Signs of cardiac tamponade are late, hence, the diagnosis of this potentially fatal condition, should be confirmed by echocardiography based on subtle clinical signs. Cardiac tamponade may occur before significant clinical signs develop. Therefore, an early echocardiography on clinical suspicion should be done to detect early 'compensated' stage of pericardial effusions (4).

In ward ultrasound scan facility is not uniformly available in all hospitals in Sri Lanka and introduced only recently in tertiary care hospitals to facilitate early detection of plasma leakage in dengue patients.Bedside ultrasound can play a crucial role in early diagnosis of certain other life threatening conditions. In our patient, the critical pericardial effusion was detected by bedside ultrasound scanning performed by junior doctors who had no formal training on radiology. This early detection led to rapid diagnosis and referral for pericardial aspiration.

There are many reports where bedside ultrasonography has been used in screening and diagnosis of a multitude of diseases. Farsi D et al, suggested that cardiac ultrasound by trained emergency medicine residents is comparable to echocardiography performed by cardiologists and it could be used as a reliable tool and screening test for initial testing of patients suspected of cardiac abnormalities in the acute setting (5). Two nonrandomized studies concluded that non-radiologist doctors could use of portable ultrasonography, effectively, in musculoskeletal disorders after a short training session $(6,7)$. This shows that bedside ultrasonography would be widely used especially in emergencies. 


\section{Conclusions}

This case history illustrates that in the absence of typical clinical signs, bedside ultrasonography by junior doctors can detect critical pericardial effusion. In ward bedside ultrasound scan has a potential role in aiding rapid diagnosis of certain life-threatening conditions. Therefore, training junior doctors on basic ultrasound examination would be helpful in early detection of critical conditions.

\section{References}

1. Das DK. Serous effusions in malignant lymphomas: a review. Diagn Cytopathol 2006; May; 34(5):335-47.

2. Acquatella GC, Roura ET, Maury AJ, Stern RO, Acquatella H. High incidence of pericardial effusion in non-Hodgkin's lymphoma: usefulness of echocardiography. Eur J Cancer Clin Oncol 1982 Nov; 18(11): 1131-6.

3. Arthur E. Baue, William S. Blakemore. The Pericardium. The Annals of Thoracic Surgery 1972 July; 14(1): 81-106.
4. Textbook of Adult Emergency Medicine. Peter Cameron, George Jelinek, Anne-Maree Kelly. $4^{\text {th }}$ Edition. ISBN $9780702053351,9780702054389$.

5. Farsi DE. et al. Focused cardiac ultrasound (FOCUS) by emergency medicine residents in patients with suspected cardiovascular diseases. J Ultrasound 2017 May 2; 20(2): 133-8.

6. Gun C, Unluer EE, Vandenberk N, Karagoz A, Senturk GO, Oyar O. Bedside ultrasonography by emergency physicians for anterior talofibular ligament injury. $J$ Emerg Trauma Shock 2013 Jul; 6(3): 195-8.

7. O’Connell K, Bouffard AJ, Vollman A, Mercado-Young R, Sargsyan AE, Rubinfeld I, et al. Extreme musculo-skeletal ultrasound: training of non-physicians in the Arctic Circle. Critical UltrasoundJ 2011 Apr;3(1): 19-24. 